\title{
Combination of ultrasound and acousto-optical imaging using a pulsed-ultrasound scanner.
}

\author{
Emmanuel Bossy ${ }^{*} \mathrm{a}, \mathrm{b}$, Lei Sui ${ }^{\mathrm{a}}$, Todd W. Murray ${ }^{\mathrm{a}}$, Ronald A. Roy ${ }^{\mathrm{a}}$ \\ ${ }^{a}$ Department of Aerospace and Mechanical Engineering, Boston University, Boston, MA 02215 \\ ${ }^{b}$ Laboratoire d'Optique Physique, ESPCI, Paris, France
}

\begin{abstract}
The acousto-optical sensing (AOS) of a turbid medium is based on the interaction of multiply-scattered coherent laser light with an ultrasonic field. A phase-modulated photon field emanates from the interaction region and carries with it information about the acousto-optical properties of the media. Using a novel technique based on a photorefractive crystal interferometer, it is possible to detect the ultrasound-modulated optical signals generated by short ultrasound pulses. As opposed to continuous-wave $(\mathrm{CW})$ ultrasound, pulsed ultrasound directly provides resolution along the ultrasonic propagation axis. In this work, a commercial ultrasound scanner (Analogic AN2300) was used in pulse mode (5 MHz central frequency) to generate both conventional ultrasound and AO images. Gel-based highly diffusive $\left(\mu_{\mathrm{s}}^{\prime}=10 \mathrm{~cm}^{-1}\right)$ tissue-mimicking phantoms were fabricated, with embedded targets possessing acoustical and/or optical contrast. AO images of 26-mm thick phantoms were generated from optical signals averaged in the time-domain, without further signal processing, and were superimposed on the top of the ultrasound images. Good quality AO images of optical absorbers, intrinsically co-registered with the ultrasound images, were obtained within minutes. The axial resolution of the AO images was given by the spatial length of the ultrasound pulse, typically on the order of one $\mathrm{mm}$ in the $\mathrm{MHz}$ range. These results show that AOI signals can be excited in pulse mode using a commercial scanner, and combined to conventional ultrasound images to provide more information related to the optical properties of the medium.
\end{abstract}

Keywords: Acousto-optical imaging, photorefractive crystal, pulsed ultrasound, diffusive media

\section{INTRODUCTION}

A number of techniques aimed at imaging the optical properties of biological tissue have been introduced recently ${ }^{1}$. Subsurface optical imaging of biological tissue is complicated by the fact that the media is highly scattering, making it difficult to achieve good spatial resolution at large imaging depths. In this context, acousto-optical sensing (AOS) shows promise, for it is based on the combination of light and ultrasound. ${ }^{2}$ Briefly, ultrasound is used to phase modulate or "tag" diffuse light, and the detection of the modulated light yields spatially resolved optical information. The spatial resolution of the underlying imaging technique, further referred to as acousto-optical imaging (AOI), is dictated by the ultrasound distribution within the media. For focused beams at $\mathrm{MHz}$ frequencies, this resolution is limited to a volume with length scales the order of a millimeter, depending on the spatial pulse length. To date, several variations of acoustooptical sensing have been introduced, based on different optical detection schemes, different types of ultrasonic waveforms (continuous, chirped or pulsed waves) and different signal processing techniques. ${ }^{2-8}$ Pulsed ultrasound is a well established clinical imaging technique, yielding information on the bulk mechanical and interfacial properties of the medium ${ }^{9}$. AOI has the potential to augment the existing technology by generating additional information regarding spatially dependent optical properties. The idea of combining information from ultrasound and AOS was first proposed by Leveque-Fort $e t$ al. ${ }^{10}$, who employed $\mathrm{CW}$ ultrasound to generate $\mathrm{AO}$ signals ${ }^{11}$. While $\mathrm{CW}$ ultrasound supports narrow bandwidth optical detection systems, and subsequent enhancement in signal-to-noise ratio (SNR), it affords limited axial resolution and can produce deleterious thermal effects on biological tissue. On the other hand, short-duration ultrasonic pulses are desirable provided that SNR limitations can be overcome. Short-pulse ultrasound yields axial resolution that is equal to the spatial pulse length, while employing low time-averaged acoustic power levels. Using a succession of phased-synchronized ultrasound pulses, Lev et al. were able to map the photon density in vitro using a tissue phantom. ${ }^{6}$ More recently, a photorefractive crystal (PRC) based interferometry system has been introduced by Murray et al. ${ }^{12}$, allowing for the detection of $\mathrm{AO}$ interaction within highly diffuse media using pulsed ultrasound. This group then showed that the use of pulsed ultrasound provides a simple way to image optical inhomogeneities along the ultrasonic axis using time-averaged AO signals ${ }^{13}$. This technique is further developed in a companion paper published in the 
present volume ${ }^{14}$, in which two-dimensional imaging is demonstrated by scanning a single-element transducer in one dimension. The use of short ultrasound pulses makes this AOI technique adaptive to conventional diagnostic ultrasound, as was recently demonstrated experimentally ${ }^{15}$. The objective is to use AOI to augment information generated by conventional B-Mode ultrasound imaging. In this paper, imaging both acoustic and AO properties using pulsed ultrasound generated by a clinical US scanner is further investigated quantitatively, in particular in terms of resolution and measurement time.

\section{MATERIALS AND METHOD}

\subsection{Experimental setup}

The experimental setup is given in Figure 1. It combines a PRC-based optical detection system ${ }^{12}$ with a commercially available, PC-based, diagnostic ultrasound scanner (AN2300, Analogic, Peabody, MA, USA). An $80 \mathrm{~mW}$ frequencydoubled Nd:YAG laser source is sent to a variable beam splitter where it is split into signal and reference beams. The reference beam is directed around the test tank and sent to the PRC-based interferometer. The signal beam is sent through a 10x beam expander along the $\mathrm{Y}$-axis to the submerged tissue-mimicking phantom, and light scattered from the phantom is collected by a lens and directed into the PRC, where it interferes with the reference beam. The PRCinterferometer employs a BSO crystal with dimensions $5 \times 5 \times 7 \mathrm{~mm}^{3}(\mathrm{X}, \mathrm{Z}, \mathrm{Y})$. A $4 \mathrm{kHz}$ high voltage field $\left(10^{6} \mathrm{~V} / \mathrm{m}\right.$ peak to peak) is applied to the crystal to enhance the two-wave mixing process. The signal beam and diffracted reference beam exiting the crystal are collected by an avalanche photodiode (APD) with a 10-mm diameter active aperture. The signal from the APD is sent to the preamplifier (x10), low-pass filtered ( $<500 \mathrm{kHz})$, and digitized by the oscilloscope. Both single shot and time-averaged waveforms can be displayed directly on the scope or transferred to the ultrasound scanner via a GPIB interface. A commercially available ultrasound probe (192-element linear array, type 8802, B\&K Medical, Herlev, Denmark) is excited by the AN2300 and projects $5 \mathrm{MHz}$ ultrasonic pulses along the Z-axis to generate both ultrasound and AO images in the ZX-plane.

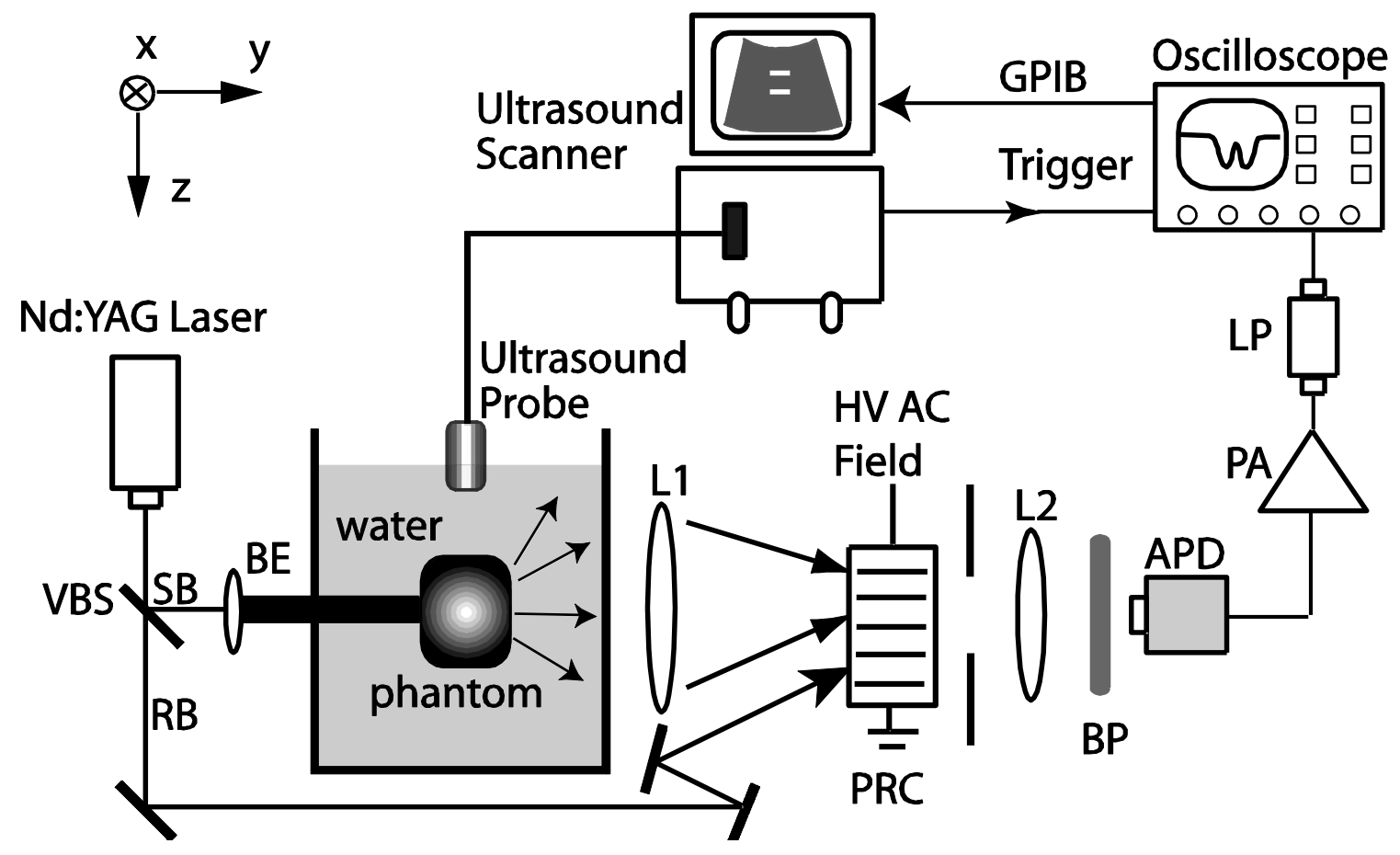

Figure 1: Experimental setup combining a PRC based interferometer with the commercial pulsed-ultrasound scanner: VBS- variable beamsplitter, RB- reference beam, SB- signal beam, BE- beam expander, UP- ultrasound probe, L1, L2- lenses, PRC- photorefractive crystal, BP- optical bandpass filter, APD- avalanche photodiode, PA- preamplifier, LP- lowpass filter. 


\subsection{Diffusive tissue phantoms}

The results reported in this paper were obtained on diffusive tissue-mimicking phantoms. The diffusive phantoms consist of a transparent polyacrylamide gel seeded with $0.4-\mu \mathrm{m}$ diameter polystyrene microspheres to obtain a reduced scattering coefficient $\mu_{\mathrm{s}}{ }^{\prime} \sim 10 \mathrm{~cm}^{-1}$. The phantoms dimensions are 40x40x26 mm (along directions $\mathrm{X}, \mathrm{Z}$ and $\mathrm{Y}$ ). A photograph of a typical phantom is shown on Fig.2(a). During the manufacturing process, targets were embedded in the phantoms using dedicated molds. The targets are made of the same material as the whole phantom, with India ink added to increase the optical absorption coefficient while minimally altering the acoustical properties. The photograph on Fig.2(b) shows a cut-away view of an optically absorbing target embedded in a diffusive phantom. Targets of various sizes were made. In the Y-direction, the targets were approximately $8 \mathrm{~mm}$ long, centered within the phantom (Fig.2). The targets were also centered in the XZ-plane, with cross-sections varying from $2 \times 2 \mathrm{~mm}^{2}$ to $5 \times 5 \mathrm{~mm}^{2}$.

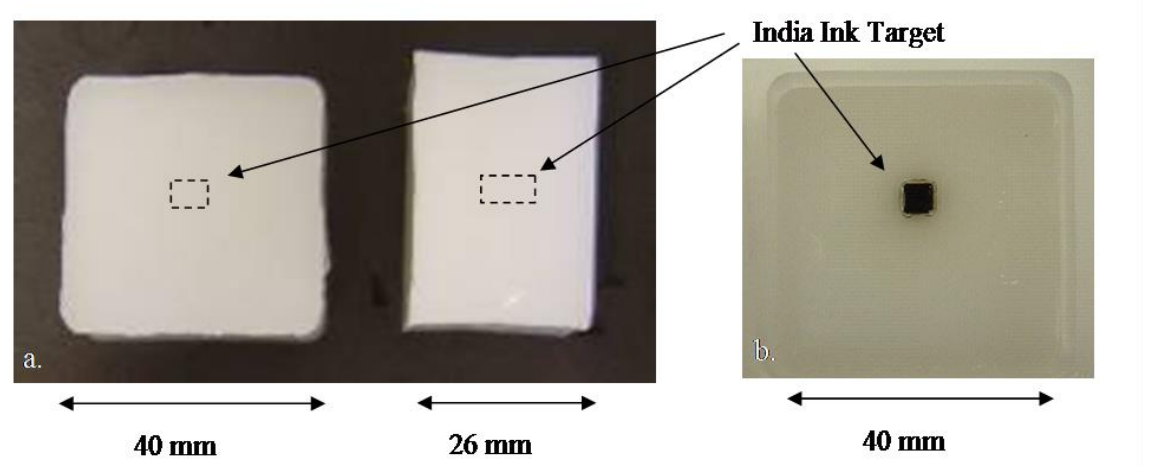

Figure 2: (a) Photograph of a highly diffusive phantom $\left(\mathrm{u}_{\mathrm{s}}^{\prime} \sim 10 \mathrm{~cm}^{-1}\right)$. The dotted rectangles indicate the position of the optical absorber shown on the cut-away view (b).

\subsection{Measurements principle}

Different approaches and models have been proposed to describe the effects of the ultrasound field on the diffuse laser light. These models consider the modulation of the optical phase induced along the different light travel paths by the ultrasound field, resulting mainly from the acoustic displacements of the light scatterers and/or acoustic modulation of the index of refraction. Independently of the mechanisms involved, we only assume here that optical phase shifts are induced along the travel paths passing through the ultrasound field. Several detection schemes have been proposed to detect this acousto-optical (AO) interaction. The experimental setup shown in Fig.1 makes use of a photorefractive crystal (PRC) based interferometry system recently introduced by Murray et al. ${ }^{12}$ for the detection of AO signals within highly diffuse media using pulsed ultrasound. The operation of the PRC-based optical detection system with pulsed ultrasound has been described elsewhere ${ }^{12,13}$. For sake of clarity, we briefly discuss the origin and nature of the detected AO signals. Without ultrasound, the signal and reference beams interfere at the PRC, and this intensity grating is recorded in the PRC as an index grating through the photorefractive effect. The reference beam diffracts from the grating into the signal beam direction with a phase front matched to the signal beam. The signal and diffracted reference beams are in-phase and interfere constructively at the APD. When an ultrasound pulse is sent through the diffusive medium, part of the scattered signal beam is phase modulated at the location of the traveling ultrasound pulse. Assuming that the duration of the $\mathrm{AO}$ interaction remains short compared to the response time of the crystal, the index grating in the PRC remains stationary, and the diffracted reference beam is no longer in phase with the transmitted signal beam. As a result of the AO interaction, a decrease of the DC output is observed at the APD. For the highly diffusive phantom used in these experiments, no AC modulation at the ultrasound frequency ( $5 \mathrm{MHz})$ is observed on the APD output. Note that the decrease of the DC output is a transient signal, short compared to the PRC response time, measured only when the spatially localized ultrasound pulse is located within the detectable diffused light field. Therefore, if one assumes that the ultrasound spatial pulse length is small compared to the spatial variation of the photon number density along the ultrasound path, the time-domain signal output at the APD is expected to reflect the distribution of detectable modulated photons along the ultrasound path.

Before discussing the experimental results that demonstrate the expected behavior described above, let us detail how the ultrasound and $\mathrm{AO}$ images were constructed. To ultrasonically scan the XZ-plane, different groups of elements on the 
probe are successively activated and fired in concentric directions, with a fixed focal length $(50 \mathrm{~mm})$. This electronic steering procedure yields a set of ultrasonic scan lines adjacent to each other. Along the direction of ultrasonic propagation, time is converted to space using an assumed sound speed $(1.5 \mathrm{~mm} / \mu \mathrm{s}$ for our water-matched phantoms). To display grayscale images (B-mode image), the ultrasound scanner demodulates the received ultrasound echo train associated with a given scan line and converts the signal envelope function to grayscale. Because the envelop of an AO signal in the time-domain is expected to correlate directly with the photon distribution along the ultrasound path, AO images were built in the exact same manner, and superimposed on top of the B-mode images. As a consequence, B-mode and $\mathrm{AO}$ images were automatically co-registered. To generate the ultrasound images, short $5 \mathrm{MHz}$ Gaussian pulses (about 1.5-cycles long) were used. Whereas a single ultrasonic shot can be used to build one B-mode line, the low SNR requires that high pressure levels, longer pulses, and time-averaging be used to detect $\mathrm{AO}$ signals. $5 \mathrm{MHz}$ sine bursts containing several cycles (typically 5-6 cycles) were used to generate the AO signals. The spatial peak, temporal peak (SPTP) excursion of the sine bursts was typically $-2 \mathrm{MPa}$ peak negative and $+4 \mathrm{MPa}$ peak positive. The pulse repetition frequency was on the order of $1 \mathrm{kHz}$ and each $\mathrm{AO}$ signal was coherently averaged over typically several thousand realizations to obtain a satisfactory SNR, as discussed in further detail in section 3.3.

\section{RESULTS AND DISCUSSION}

\subsection{Combination of B-Mode and $\mathrm{AO}$ images}
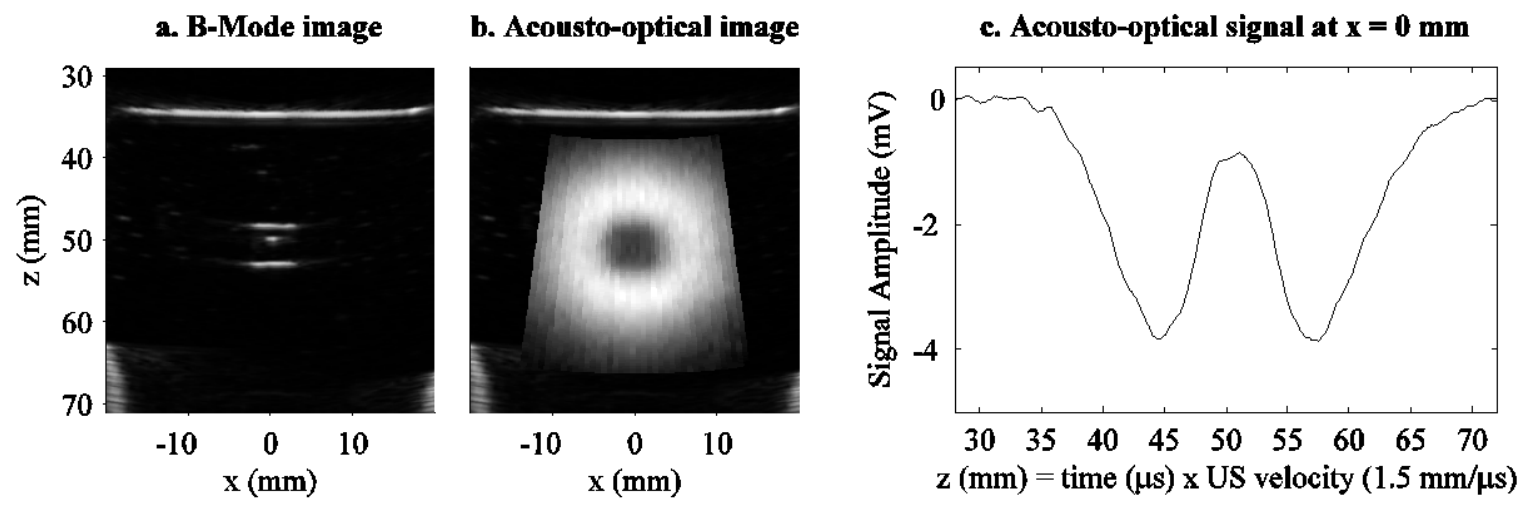

Figure 3: B-mode (a) and $\mathrm{AO}$ (b) images of a 5x5x8 $\mathrm{mm}^{3}$ (z,x,y) optical absorbers. (c) A typical time averaged AO signal as displayed on the oscilloscope for a single ultrasound scan line traversing the absorber $(\mathrm{x}=0 \mathrm{~mm})$.

Figure 3 shows the B-Mode image (a) and superimposed AO image (b) obtained from a phantom with a $5 \times 5 \times 8 \mathrm{~mm}^{3}$ optical absorber. Five-cycle 5-MHz acoustic pulses (spatial length of about $1.5 \mathrm{~mm}$ ) were used. For each scan line, AO signals were averaged 25,000 times. The field of view was matched to the ZX dimensions of the phantoms. On the BMode image, the bright line observed at $\mathrm{z}=34 \mathrm{~mm}$ corresponds to the specular ultrasonic reflection on the proximal interface of the phantom, due to the slight impedance mismatch between the phantom and the surrounding water bath. The bright regions located in both distal corners correspond to the holder used to maintain the phantom still in the water. The absorbing target itself is barely visible in the ultrasound image, as expected for such a low contrast inhomogeneity. No acoustic contrast is seen within the absorber. The parallel horizontal lines that demarcate the proximal and distal interfaces of the absorber are due largely to imperfect bonding of the target and phantom materials during the manufacturing process. A typical AO signal obtained for an ultrasound path through the absorber is plotted in Fig. 3(c). The AO image has a field of view given by the circular region seen on Fig.3(b), typically $20 \mathrm{~mm}$ in diameter. This distribution of detected modulated light is limited by the light distribution itself, but may also be limited by the aperture and position of the light collecting system. In all experiments, the phantoms were positioned so that the absorber is located at the center of the light distribution and at the ultrasound focus $(\mathrm{z}=50 \mathrm{~mm})$, in order to optimize SNR and resolution. Both the lateral $(\mathrm{X})$ and axial $(\mathrm{Z})$ dimensions on the AO image visually agree with the actual dimension of the absorber $(5 \mathrm{~mm} \times 5 \mathrm{~mm})$. Quantitatively, the width at half maximum measured from Fig.3(c) along the axial dimension yields a value of approximately $5 \mathrm{~mm}$, in agreement with the actual dimension of the absorber. This illustrates the ability 
of this pulsed-ultrasound technique to obtained millimeter resolution along the axial dimension. As further discussed in the next section, the axial resolution is dictated by the spatial acoustic pulse length.

\subsection{Resolution of the $\mathrm{AO}$ images}

As the AO signals originate from the region where both light and ultrasound are simultaneously present to interact, it is expected that the ultrasonic resolution is limited by the ultrasonic pulse spatial extension. This has been demonstrated experimentally for the lateral resolution, in the directions perpendicular to the ultrasound beam ${ }^{3,16}$. For our 5-MHz probe, the lateral resolution in the imaging plane (X direction) defined by the full-width-at-half-maximum intensity (FWHM) was typically $0.7-0.8 \mathrm{~mm}$. As illustrated in the previous section, using pulsed-ultrasound also allows for improved axial resolution. Figure 4(a) shows AO images of a $5 \times 5 \times 8 \mathrm{~mm}^{3}$ optical absorber obtained for different pulses of varying length. The ultrasound frequency is fixed at $5 \mathrm{MHz}$, the pulse length is controlled by the pulse duration through varying the number of cycles ( 4 cycles for $1.2 \mathrm{~mm}, 40$ cycles for $12 \mathrm{~mm}$ ). A typical acoustic pulse, measured with a calibrated hydrophone at the focus, is shown on Fig.4(b). The distorted shape of the pulse is caused by non-linear propagation effects consequent to the relatively high amplitude at the focus.

\section{a. Acousto-optical images obtained for different pulse lengths}
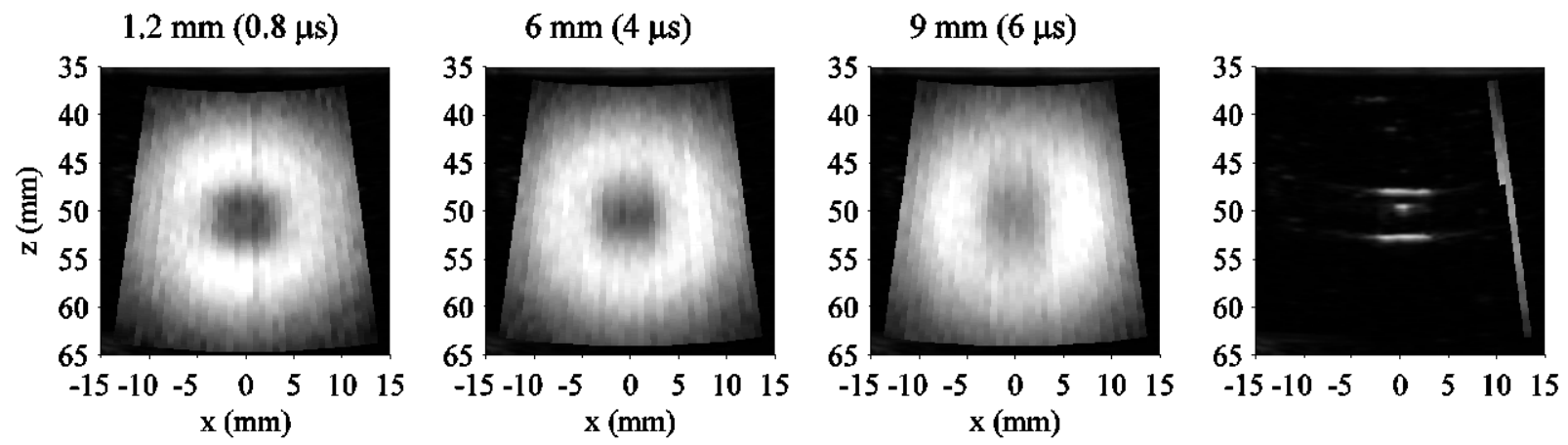

Figure 4: (a) OA images of a 5x5x8 $\mathrm{mm}^{3}$ optical absorber, obtained for different acoustic pulse lengths (durations). (b) Typical acoustic pulse, measured with a calibrated hydrophone at the focus. (c) Acousto-optical signals measured when the ultrasound pulse travels along a path going through the optical absorber, for different pulse lengths.

The AO images show that axial resolution clearly depends on the pulse length, as expected from the discussion in 2.3. For a 12-mm long pulse, i.e. slightly more than twice the absorber size along the axial dimension, axial resolution is completely lost, and the absorber is only made detectable through the scan along the transverse dimension of the absorber. As shown on both the AO images (Fig.4(a)) and the AO signals measured for a line going through the absorber (Fig.4(c)), the contrast along the axial dimension increases as the pulse length decreases. In principle, the contrast is expected to be 1 (no signal at all within the absorbing target) if the ultrasound pulse is spatially small enough to be confined to the absorber only. Whereas it should be the case for a pulse length of $1.2 \mathrm{~mm}$, it is likely that lateral side 
lobes in both the $\mathrm{X}$ and $\mathrm{Y}$ directions are responsible for some $\mathrm{AO}$ interaction outside the absorber, yielding residual $\mathrm{AO}$ signal as if it resulted from within the absorber. Using a 3-MHz (type 8665, B\&K Medical) and a (12-MHz type 8805, $\mathrm{B} \& \mathrm{~K}$ Medical) probe (results not shown here), we verified that the axial resolution is dictated only by the pulse length, independently of the frequency. The ability of this AO imaging technique to detect absorbers of various sizes is illustrated on Fig.5. 3-cycle 5-MHz pulses (spatial length about $1 \mathrm{~mm}$ ) were used. The 2x2x8 $\mathrm{mm}^{3}$ optical absorber is imaged with a good contrast close to its actual size.
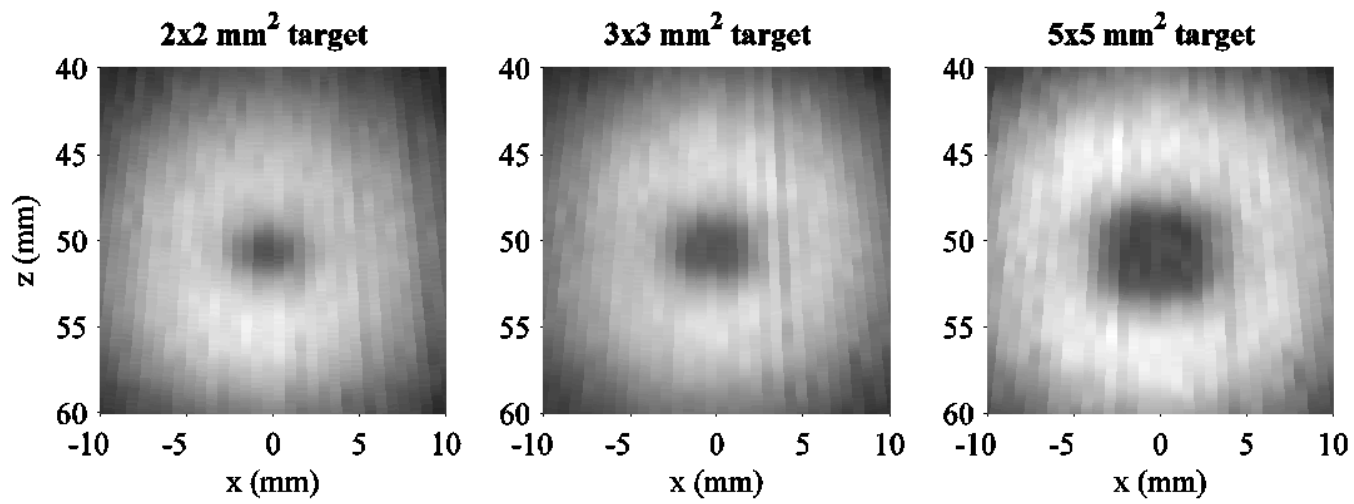

Figure 5: AO images of three different phantom, with optical absorbers of various size $\left(2 \times 2 \times 8 \mathrm{~mm}^{3}, 3 \times 3 \times 8 \mathrm{~mm}^{3}\right.$ and $5 \times 5 \times 8 \mathrm{~mm}^{3}$, from left to right). 3-cycle 5-MHz pulses (spatial length about $1 \mathrm{~mm}$ ) were used for all three measurements.

Figure 6 illustrates the ability of the technique to resolve two absorbers within the same phantom. The phantom, containing two $3 \times 3 \times 8 \mathrm{~mm}^{3}$ absorbers $3 \mathrm{~mm}$ apart, was rotated to illustrate both axial and lateral resolution.

\section{a. Lateral resolution}

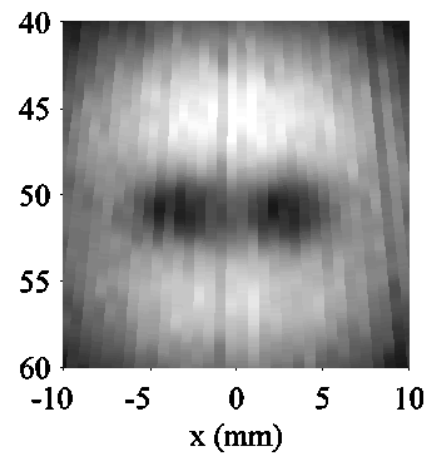

b. Axial resolution

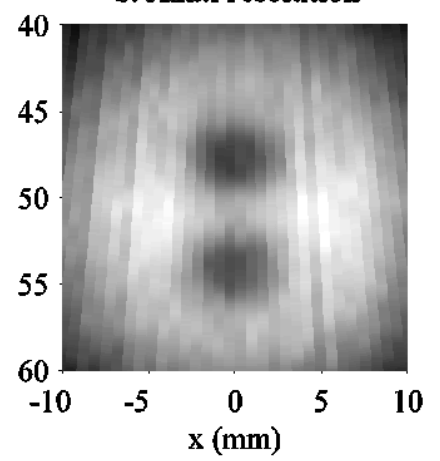

Figure 6: AO images of two 3x3x8 $\mathrm{mm}^{3}(\mathrm{z}, \mathrm{x}, \mathrm{y})$ identical optical absorbers separated by $3 \mathrm{~mm}$. (a) The two targets were aligned perpendicularly to the ultrasound axis. (b) The phantom was then rotated to align the two targets along the ultrasound axis.

Emmanuel, the lateral blurring effect in Figure 6(a) in comparing with Figure 6(b), I think, is due to the side lobe effect because axial resolution is better than lateral. Of course, however, I think that the "Shadow Effect" (high possibility for photons nearby the absorber to be absorbed) is also responsible for part of it.

\subsection{SNR and measurement time}

In our PRC based detection scheme, transient AO signals are recorded and averaged coherently many times to increase SNR. The absolute amplitude of one single AO signal (without averaging) depends on many parameters, such as the laser power, the acoustic pulse duration, the acoustic pulse amplitude. The amplitude of the noise is independent of the ultrasound field, and is at best limited by the shot noise at the APD. For a fixed optical parameters (lased power, gain on the APD, etc.), the SNR for a single signal is dependent only on the properties of the acoustic pulse. Therefore, the 
amplitude of the acoustic pulse should be as high as possible. In the context of in vivo breast imaging for instance, the acoustic energy is of course limited by safety consideration. The spatial-peak pulse-average intensity ( $\left.\mathrm{I}_{\mathrm{SPPA}}\right)$ of the acoustic pulse that was used in this work was typically $200 \mathrm{~W} / \mathrm{cm}^{2}$, and corresponds approximately to the maximum value allowed by the U.S. Foods and Drug Administration (FDA) ${ }^{17}$.
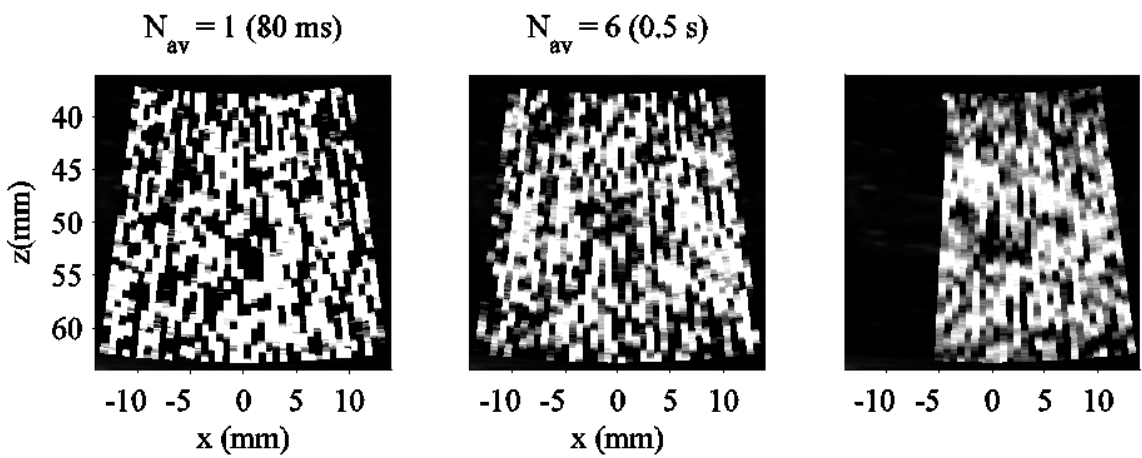

Figure 7: AO images obtained using several numbers of averages per scan line (noted N). Each image consists of 41 scan lines. The measurement times are those obtained for a PRF of $500 \mathrm{~Hz}$ and 41 lines per image, assuming the data transfer and processing times can be neglected.

The upper left AO image on Fig.7 shows the AO image obtained with one single AO signal per scan line, without coherent averaging. It clearly shows that the SNR in this case is too low to obtain a readable AO image. AO images obtained using several numbers of averages per line (noted $\mathrm{N}$ ) are shown on Figure 7, for a given acoustic pulse (5-cycle long bursts at $5 \mathrm{MHz}, \mathrm{I}_{\mathrm{SPPA}} \sim 200 \mathrm{~W} / \mathrm{cm}^{2}$ ). When the maximum amplitude of the AO signals is estimated from that obtained with coherent averaging, the SNR (defined as the ratio of the maximum signal amplitude to the rms value of the noise) obtained for a single AO signal without averaging turns out to be of the order of $1: 2$, less than 1 as seen straightforwardly on the upper left image. As a function of N, the SNR turns out to be approximately proportional to $\sqrt{ } \mathrm{N}$, as expected for coherent averaging of uncorrelated measurements. For $N \sim 20,000$, the SNR is of the order of 70:1. The particular values of the SNR as a function of $\mathrm{N}$ would of course be different for different light powers or different other optical parameters. The total measurement time for one image depends on the number of lines per image and primarily on the time per line (i.e. the acoustic pulse repetition frequency, PRF). In vivo, the PRF for the high amplitudes used here is limited by the spatial-peak time-average intensity $\left(\mathrm{I}_{\mathrm{SPTA}}\right)$. The measurement times indicated on Fig.7 are those obtained for a PRF of $500 \mathrm{~Hz}$ and 41 lines per image, assuming the data transfer and processing times can be neglected, which can be easily achieved on dedicated hardware. Such a PRF was chosen to calculate the measurement times as it gives an $\mathrm{I}_{\text {SPTA }}$ value of approximately $100 \mathrm{~mW} / \mathrm{cm}^{2}$, close to the FDA limit $\left(94 \mathrm{~mW} / \mathrm{cm}^{2}\right)$ for small organs. These measurement times are only intended here to give an order of magnitude of the necessary time to obtain good quality image, given that the laser power was rather low, but the experiments carried out in vitro. The real measurement time for our in vitro measurement was approximately ten times longer than that calculated for the used PRF of $3 \mathrm{KHz}$, as the data transfer rate and averaging through the oscilloscope and GPIB was not negligible in this case.

\subsection{Supplementing B-mode with acousto-optical imaging}


To further illustrate the ability of this technique to supplement conventional B-Mode imaging, a phantom was made with two embedded targets $\left(3 \times 3 \times 8 \mathrm{~mm}^{3}\right.$ each, separated by $\left.3 \mathrm{~mm}\right)$, one with India ink and the other without. The two targets appear identical in the B-Mode image (Fig.8(a)), which only shows interfaces but no acoustic contrast within the targets. On the other hand, the AO image (Fig.8(b)) leaves no doubt as to the differing absorbing nature of each target. In Fig.8(c), typical signals across each target are plotted, and a contrast value of approximately $75 \%$ is found. In a clinical context, this suggests the ability to distinguish between optically distinct clinical features ${ }^{18,19}$ that would otherwise appear identical on a conventional ultrasound image.
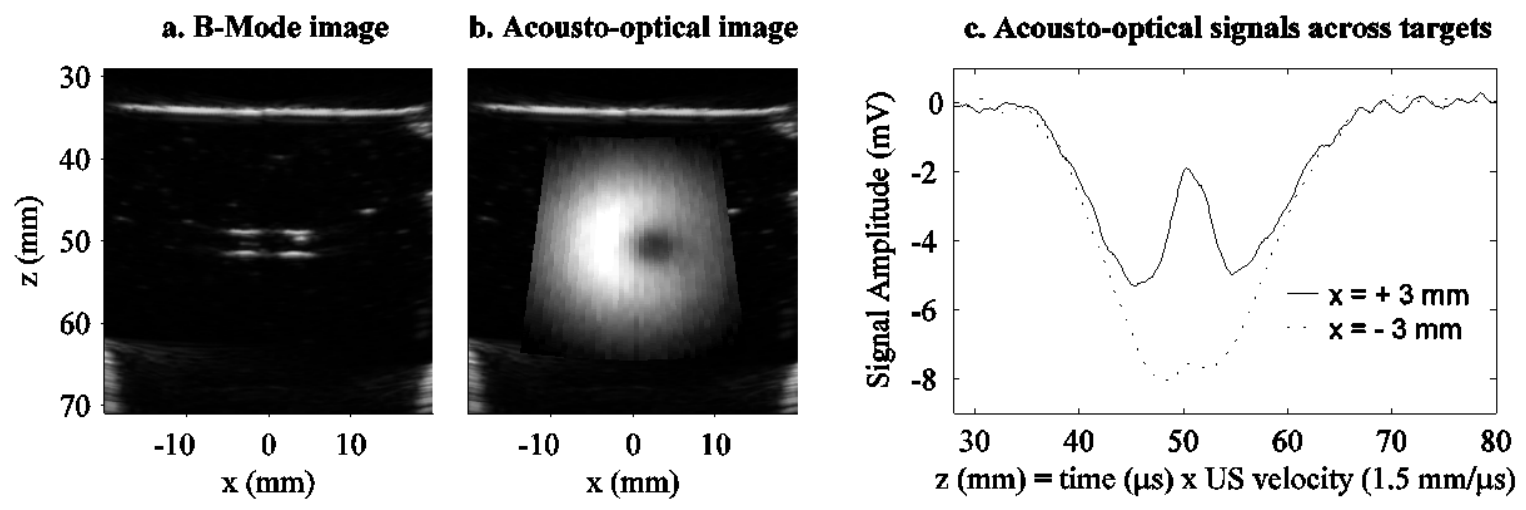

Figure 8: B-mode (a) and $\mathrm{AO}$ (b) images of two $3 \times 3 \times 8 \mathrm{~mm}^{3}(\mathrm{z}, \mathrm{x}, \mathrm{y})$ targets separated by $3 \mathrm{~mm}$ along the $\mathrm{x}$ axis. The target at $\mathrm{x}=-3$ $\mathrm{mm}$ is identical to the background medium, whereas the target at $\mathrm{x}=+3 \mathrm{~mm}$ is absorbing. (c) Typical time averaged AO signals as displayed on the oscilloscope for separate scan lines traversing across each target.

\section{CONCLUSIONS}

These experimental results reported herein demonstrate in vitro that B-mode and $\mathrm{AO}$ sensing can be simultaneously implemented using a commercially available ultrasound scanner, yielding both acoustical and optical contrast information in the form of two automatically co-registered images. Deleterious bio-effects of high intensity $\mathrm{CW}$ ultrasound are avoided, and using short pulses to excite the AO response enables the utilization of standard imaging technology to yield a true multi-mode imaging capability sensitive to both acoustical and optical contrast. A standard 5 $\mathrm{MHz}$ ultrasound imaging probe was used to excite the AO signals, and images were formed with no additional signal processing other than that already implemented on clinical machines. Good quality AO images of highly diffusive phantoms $\left(\mu_{s}^{\prime}=10 \mathrm{~cm}^{-1}\right)$ were obtained with millimeter axial and lateral resolution within minutes, demonstrating fast acousto-optical imaging. The detection of pulse modulated light was facilitated by the use of a PRC-based interferometer. To demonstrate the feasibility of this technique for imaging real biological tissue in vivo, speckle decorrelation associated with tissue motion has to be overcome, requiring the PRC response time to be short with respect to the speckle decorrelation time in vivo. A crystal possessing a response time faster than the BSO crystal used in our setup (approximately $150 \mathrm{~ms}$, requiring some degree of mechanical isolation) must be employed before moving on to in vivo studies.

\section{ACKNOWLEDGEMENT} 9986821.

This work was supported by the Center for Subsurface and Imaging Systems via NSF ERC award number EEC-

\section{REFERENCES}

1. V. V. Tuchin, Handbook of Optical Biomedical Diagnostics (SPIE, Washington, 2002).

2. L. V. Wang, "Ultrasound-mediated biophotonic imaging: a review of acousto-optical tomography and photoacoustic tomography," Dis Markers 19(2-3), 123-138 (2003).

3. L. V. Wang, S. L. Jacques, and X. M. Zhao, "Continuous-Wave Ultrasonic Modulation of Scattered Laser-Light to Image Objects in Turbid Media," Optics Letters 20(6), 629-631 (1995). 
4. M. Kempe, M. Larionov, D. Zaslavsky, and A. Z. Genack, "Acousto-optic tomography with multiply scattered light," Journal of the Optical Society of America a-Optics Image Science and Vision 14(5), 1151-1158 (1997).

5. S. Leveque, A. C. Boccara, M. Lebec, and H. Saint-Jalmes, "Ultrasonic tagging of photon paths in scattering media: parallel speckle modulation processing," Optics Letters 24(3), 181-183 (1999).

6. A. Lev and B. G. Sfez, "Pulsed ultrasound-modulated light tomography," Optics Letters 28(17), 1549-1551 (2003).

7. B. C. Forget, F. Ramez, M. Atlan, J. Selb, and A. C. Boccara, "High-contrast fast Fourier transform acoustooptical tomography of phantom tissues with a frequency-chirp modulation of the ultrasound," Applied Optics 42(7), 1379-1383 (2003).

8. M. Gross, P. Goy, and M. Al-Koussa, "Shot-noise detection of ultrasound-tagged photons in ultrasoundmodulated optical imaging," Optics Letters 28(24), 2482-2484 (2003).

9. T. L. Szabo, Diagnostic Ultrasound Imaging: Inside Out (Elsevier Academic Press, Boston, 2004).

10. S. Leveque-Fort, J. Selb, L. Pottier, and A. C. Boccara, "Towards simultaneous acousto-optical and acoustical imaging in biological tissues," presented at the Photon Migration, Diffuse Spectroscopy, and Optical Coherence Tomography: Imaging and Functional Assesment, 2000.

11. J. Selb, S. Leveque-Fort, L. Pottier, and A. C. Boccara, "Setup for simultaneous imaging of optical and acoustic contrasts in biological tissues," presented at the Biomedical Optoacoustics II, San Jose, CA, USA, 2001.

12. T. W. Murray, L. Sui, G. Maguluri, R. A. Roy, A. Nieva, F. Blonigen, and C. A. DiMarzio, "Detection of ultrasound-modulated photons in diffuse media using the photorefractive effect," Optics Letters 29(21), 25092511 (2004).

13. L. Sui, R. A. Roy, C. A. DiMarzio, and T. W. Murray, "Imaging in diffuse media using pulsed ultrasoundmodulated light and the photorefractive effect.," Applied Optics (accepted for publication).

14. L. Sui, R. A. Roy, C. A. DiMarzio, F. Blonigen, and T. W. Murray, "Investigation of the photorefractive crystal based detection system for acousto-optical imaging (AOI) in highly diffuse media," presented at the SPIE, San Jose, CA, To be published.

15. E. Bossy, L. Sui, T. W. Murray, and R. A. Roy, "Fusion of conventional ultrasound imaging and acousto-optical sensing (AOS) using a standard pulsed ultrasound scanner," Optics Letters (in press).

16. S. Leveque-Fort, "Three-dimensional acousto-optic imaging in biological tissues with parallel signal processing," Applied Optics 40(7), 1029-1036 (2001).

17. http://www.fda.gov/cdrh/ode/ulstran.pdf, "Information for manufacturers seeking marketing clearance of diagnostic ultrasound systems and transducers," (U.S. Department of Health and Human Services, Food and Drug Administration, Center for Devices and Radiological Health, Rockville, 1997).

18. B. J. Tromberg, N. Shah, R. Lanning, A. Cerussi, J. Espinoza, T. Pham, L. Svaasand, and J. Butler, "Noninvasive in vivo characterization of breast tumors using photon migration spectroscopy," Neoplasia 2(1-2), 2640 (2000).

19. J. B. Fishkin, O. Coquoz, E. R. Anderson, M. Brenner, and B. J. Tromberg, "Frequency-domain photon migration measurements of normal and malignant tissue optical properties in a human subject," Applied Optics 36(1), 10-20 (1997). 